\title{
A randomised phase II/III trial of 3-weekly cisplatin-based sequential transarterial chemoembolisation vs embolisation alone for hepatocellular carcinoma
}

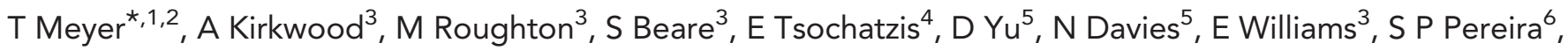
D Hochhauser ${ }^{2}$, A Mayer $^{1}$, R Gillmore ${ }^{1}$, J O'Beirne ${ }^{4}$, D Patch ${ }^{4}$ and A K Burroughs ${ }^{4}$

${ }^{1}$ Department of Oncology, UCL Medical School, Royal Free Campus, London NW3 2QG, UK; ${ }^{2}$ UCL Cancer Institute, 72 Huntley Street, London WC1E 6BT, UK; ${ }^{3}$ Cancer Research UK \& UCL Cancer Trials Centre, London W1T 4TJ, UK; ${ }^{4}$ The Royal Free Sheila Sherlock Liver Centre and University Department of Surgery and Interventional Science, Royal Free London NHS Foundation Trust, London NW3 2QG, UK; ${ }^{5}$ Department of Radiology, Royal Free London NHS Foundation Trust, London, UK and ${ }^{6}$ Department of Gastroenterology, University College Hospital, London NW1 2BU, UK

Background: Transarterial chemoembolisation (TACE) has not been shown to be superior to bland embolisation (TAE) for treatment of hepatocellular carcinoma (HCC).

Methods: We conducted a randomised phase II/III trial in patients with untreated HCC. Patients were randomised to TAE with polyvinyl alcohol (PVA) particles alone or sequential TACE (STACE) in which cisplatin $50 \mathrm{mg}$ was administered intrarterially 4-6h before PVA embolisation. Treatment was repeated 3-weekly for up to three treatments. The primary endpoint was overall survival and secondary endpoints were progression-free survival, toxicity and response. Target sample sizes for phase II and III were 80 and 322 .

Results: The trial was terminated at phase II after 86 patients had been randomised. Patients were well matched for prognostic criteria. All three planned treatments were given to $57.1 \%$ (TAE) and $56.8 \%$ (TACE) patients. Grade 3/4 toxicity occurred in $63.5 \%$ and $83.7 \%$, respectively $(P=0.019)$. End-of-treatment RECIST response (CR $+P R)$ was 13.2 and $32.6 \%(P=0.04)(\mathrm{mRECIST} 47.3 \%$ and 67.4) and median overall survival and progression-free survival was 17.3 vs $16.3(P=0.74)$ months and 7.2 vs $7.5(P=0.59)$, respectively.

Conclusion: Transarterial chemoembolisation according this novel schedule is feasible and associated with a higher response rate than TAE alone. The survival benefit of TACE over TAE remains unproven.

Hepatocellular carcinoma (HCC) is the sixth most common type of cancer worldwide and the third most common cause of death from cancer, resulting in around 600000 deaths annually (Parkin et al, 2005). The major risk factors are infection with hepatitis $\mathrm{B}$ and $\mathrm{C}$ virus, which increase the risk of liver cancer 20 -fold. Liver cirrhosis is present in $80 \%$ of cases and both prognosis and treatment are determined by liver function as well as tumour-related factors and performance status (Okuda et al, 1985; CLIP Investigators, 1998; Llovet et al, 1999). Overall the prognosis is extremely poor with incidence closely matching mortality. Potentially curative options including transplantation, resection or ablation are applicable to less than $30 \%$ of patients who meet defined criteria and the majority of patients are therefore treated with palliative intent. 
Transarterial embolisation (TAE) and chemoembolisation (TACE) have been widely used to treat HCC and exploit the dual blood supply to the liver in which the liver parenchyma receives most of its blood supply from the portal vein, while the tumour is supplied predominantly by the hepatic artery. This allows selective cannulation of vessels supplying the tumour and occlusion by embolic particles leading to tumour hypoxia and necrosis. The procedure can be performed with the co-administration of arterial chemotherapy (transarterial chemoembolisation, TACE), but there is considerable heterogeneity in the technique in relation to the type of embolic particle, the chemotherapy drugs used and the schedule of administration (Marelli et al, 2007). Evidence that TACE improves survival comes from two small randomised trials in which gelfoam embolisation was performed with either doxorubicin (Llovet et al, 2002) or cisplatin (Lo et al, 2002) and found to be superior to best supportive care. These trials were included in a subsequent meta-analysis of seven trials including 545 patients, which concluded that TACE, but not TAE, was associated with a significant 2-year survival benefit over best supportive care (Llovet and Bruix, 2003). Since the publication of this meta-analysis two further trials have been reported and an updated meta-analysis performed by the Cochrane Collaboration which included nine trials and 645 participants (Oliveri et al, 2011). It was observed that the individual trials were underpowered, at risk of bias and that there was heterogeneity between the trials. Overall, the authors concluded neither TAE nor TACE improved survival compared with best supportive care. The discrepancy between these two reviews is attributable to application of different criteria for trial inclusion and the validity of the Cochrane review has been questioned on this basis (Ray, Jr et al, 2011; Forner et al, 2012).

The contribution of chemotherapy to the effectiveness of TACE has also been questioned given that HCC is relatively chemoresistant (Burroughs et al, 2004; Asghar and Meyer, 2012), and there is currently no definite evidence that TACE is superior to TAE (Marelli et al, 2007). However, the conventional method of delivering TACE may not be optimal in terms of achieving maximum cytotoxic effect from chemotherapy. First, TACE is usually performed at intervals of several months rather than according to more frequent oncological schedules designed to prevent tumour repopulation. Second, the coadministration of chemotherapy with particles may compromise the delivery of drug to the tumour, and the resulting tumour hypoxia is known to be an important cause of chemoresistance (Tak et al, 2011; Asghar and Meyer, 2012).

Given these considerations and the uncertain benefit of TACE over TAE, we designed a randomised trial in which the delivery of chemotherapy was optimised to maximise its potential benefit. We selected cisplatin, as this drug is well tolerated in liver impairment and was effective in a previously reported randomised trial (Lo et al, 2002). Cisplatin induces DNA damage by inducing intra- and interstrand crosslinks which in turn lead to apoptosis or cell cycle arrest and DNA repair. For our study, cisplatin was administered 4-6h before embolisation allowing DNA damage to occur before acute hypoxia. We have termed this procedure sTACE (sequential TACE) to distinguish it from other types of TACE such as; (a) lipiodol-based TACE, which has been termed 'conventional' TACE (cTACE) in which TAE is performed immediately following administration of a lipiodol/cytotoxic suspension, (b) drug-eluting bead (DEB)-TACE in which chemotherapy is conjugated directly to DEBs. To reduce the opportunity for tumour repopulation, a 3-weekly treatment schedule was mandated.

\section{METHODS}

Trial Design. This phase II/III trial was designed to compare TAE with sTACE in patients with non-resectable HCC. Patients were randomised to one of the two treatment arms in a $1: 1$ ratio. Randomisation was carried out by the coordinating centre using a paper list, and was stratified by recruiting site. The purpose of the phase II aspect of the trial was to examine the safety, tolerability and feasibility of delivering the treatments.

This trial was ethically approved by the institutional review board and registered on an appropriate clinical trial databases (ISRCTN63349435). The trial was coordinated centrally by the Cancer Research UK and University College London Cancer Trials Centre (UCL CTC). Ongoing safety and efficacy were independently assessed by an Independent Data Monitoring Committee. The design, conduct and reporting of this trial adheres to the CONSORT 2010 Statement (Schulz et al, 2010).

Patients. Patients were eligible if they were $\geqslant 16$ years old and had HCC diagnosed according to the EASL criteria (Bruix et al, 2001); (1) histology or (2) evidence of a focal lesion $>2 \mathrm{~cm}$ with arterial hypervascularisation detected on two radiological studies (any two of ultrasound, computed tomography (CT), magnetic resonance imaging or angiography) in a patient with a background of cirrhosis; or (3) a single radiological study with an $\alpha$-fetoprotein greater than $400 \mathrm{ng} \mathrm{ml}^{-1}$. Patients were not eligible if they were candidates for surgical resection. The extent of disease was required to be either a solitary hepatic tumour $>3 \mathrm{~cm}$ in diameter or multifocal. Other eligibility criteria included ECOG performance status (PS) $0-2$, platelets $\geqslant 50 \times 10^{9}$ per litre, $\mathrm{INR} \leqslant 1.5$, $\mathrm{GFR} \geqslant 50 \mathrm{ml} \mathrm{min}^{-1}$, bilirubin $\leqslant 100 \mu \mathrm{moll}^{-1}$. Patients were not eligible if they had received prior treatment for HCC, if there was evidence of extra-hepatic metastases, or if they had a Child Pugh score of C. Patients were also excluded if they had active sepsis or bleeding, hepatic encephalopathy, ascites refractory to diuretic therapy, or documented occlusion of the hepatic artery or portal vein. Pregnant or lactating women were not eligible for the trial.

Procedures. Patients received either TAE or sTACE. Treatment with TAE consisted of selective angiography followed by embolisation with $50-150 \mu \mathrm{m}$ polyvinyl alcohol (PVA) particles at segmental or lobar level. Bilobar lesions were treated separately if they could not be treated by highly selective (segmental or sub segmental) embolisation. Prophylactic antibiotics were administered to reduce the risk of infection. For sTACE, cisplatin $(50 \mathrm{mg}$ in $50 \mathrm{ml}$ ) was administered intra-arterially as an injection over $15 \mathrm{~min}$. The cisplatin injection was preceded by intravenous (i.v.) hydration and anti-emetics and followed by more i.v. hydration. TAE was performed as described above $4-6 \mathrm{~h}$ after cisplatin administration. Treatment was repeated up to three times at 3-weekly intervals. Patients were withdrawn from further trial treatment if treatment was delayed by more than 3 weeks due to haematological toxicity or if they experienced any grade IV nonhaematological toxicity. After the final treatment, patients were assessed at 1 month and then 3 monthly, thereafter. Further TAE procedures were undertaken at the investigator's discretion if the tumour demonstrated residual vascularity but sorafenib was not offered on progression because approval and funding only became available after the completion of the trial.

Adverse events (AEs) were assessed before each treatment, at completion of treatment and at all follow-up visits and graded according to the NCI Common Terminology Criteria for Adverse Events (CTCAE) version 3.0. Patients were invited to complete quality of life (QoL) questionnaires (EORTC QLQ-C30 and EORTC QLQ-HCC 18). QoL questionnaires were completed before start of treatment, at completion of treatment and 3 and 6 months after completion of treatment.

Before commencing treatment, patients were staged by means of a triphasic spiral CT scan of the chest and abdomen or a CT scan of the thorax and magnetic resonance imaging (MRI) of the abdomen. Repeat scanning was carried out at completion of treatment and 3 monthly thereafter until progression. Response 
was assessed according to RECIST 1.0 (Therasse et al, 2000) criteria and mRECIST (Lencioni and Llovet, 2010; Gillmore et al, 2011). Patients who initially responded were offered a further course of treatment upon progression, provided they met the treatment criteria listed above. Patients who received a liver transplant were followed-up according to the local transplant protocol.

Statistics. The recruitment target for the phase II trial was 80 patients (40 in each arm) with the aim of assessing safety, tolerability and the feasibility of delivering the treatments. We considered that an underlying grade 4 toxicity rate of greater than $30 \%$ would be unacceptable. Therefore, if 19 patients in either arm suffered any toxicity of grade 4 (except pain), the trial was to be stopped. Patients who were recruited into TACE, and then subsequently received a liver transplant, were replaced by recruiting additional patients to be able to fully evaluate treatment safety. In April 2009, the independent data monitoring committee (IDMC) were asked to advise on increasing the phase II recruitment target to compensate for patients who underwent liver transplants. The IDMC agreed that this was reasonable and the sample size for the phase II trial was increased to obtain 80 evaluable patients.

The phase III part of the trial was designed to detect a difference in 2-year survival from 50 to $63 \%$ with $80 \%$ power and a $5 \%$ level of statistical significance. For this, 322 patients would have been recruited over 4 years with follow-up of at least 1 year. In February 2010, the IDMC recommended terminating the trial because the recruitment rate was not compatible with a timely completion of the phase III trial.

Comparison of survival between the two treatment groups was made using the log-rank test and Cox regression was used to estimate the hazard ratio. We also ran models that included the site, the stratification factor used in the randomisation. The following planned subgroup analyses were carried out; Child Pugh, Okuda, Barcelona-Clínic Liver Cancer (BCLC), Cancer of the Liver Italian Program (CLIP) and French staging classifications (10). Quality of life scores were analysed by comparing changes from baseline scores between the two treatment groups.

We performed an update to our published meta-analysis comparing TAE vs TACE (Marelli et al, 2007) using as primary outcome the number of patients reported as dead during follow-up. The meta-analysis was conducted according to the PRISMA recommendations (Moher et al, 2009). Studies were identified by searching Medline and Embase using the following keywords: 'hepatocellular carcinoma' or 'HCC' or 'hepatic tumour' or 'liver tumour' or 'hepatic cancer' or 'liver cancer' and 'TACE' or 'TAE' or 'chemoembolisation' or 'embolisation' and 'clinical trials'. The analyses were performed using RevMan version 5.1 (Nordic Cochrane Centre, Copenhagen, Denmark). Meta-analyses were performed using random effects models due to expected clinical heterogeneity. The number of events and number of patients in each intervention arm were used to calculate relative risks (RR) and $95 \%$ confident intervals (CI). $I^{2}$ values were calculated as measures of the degree of intertrial heterogeneity. Data on all patients randomised were extracted to allow intention-to-treat analyses.

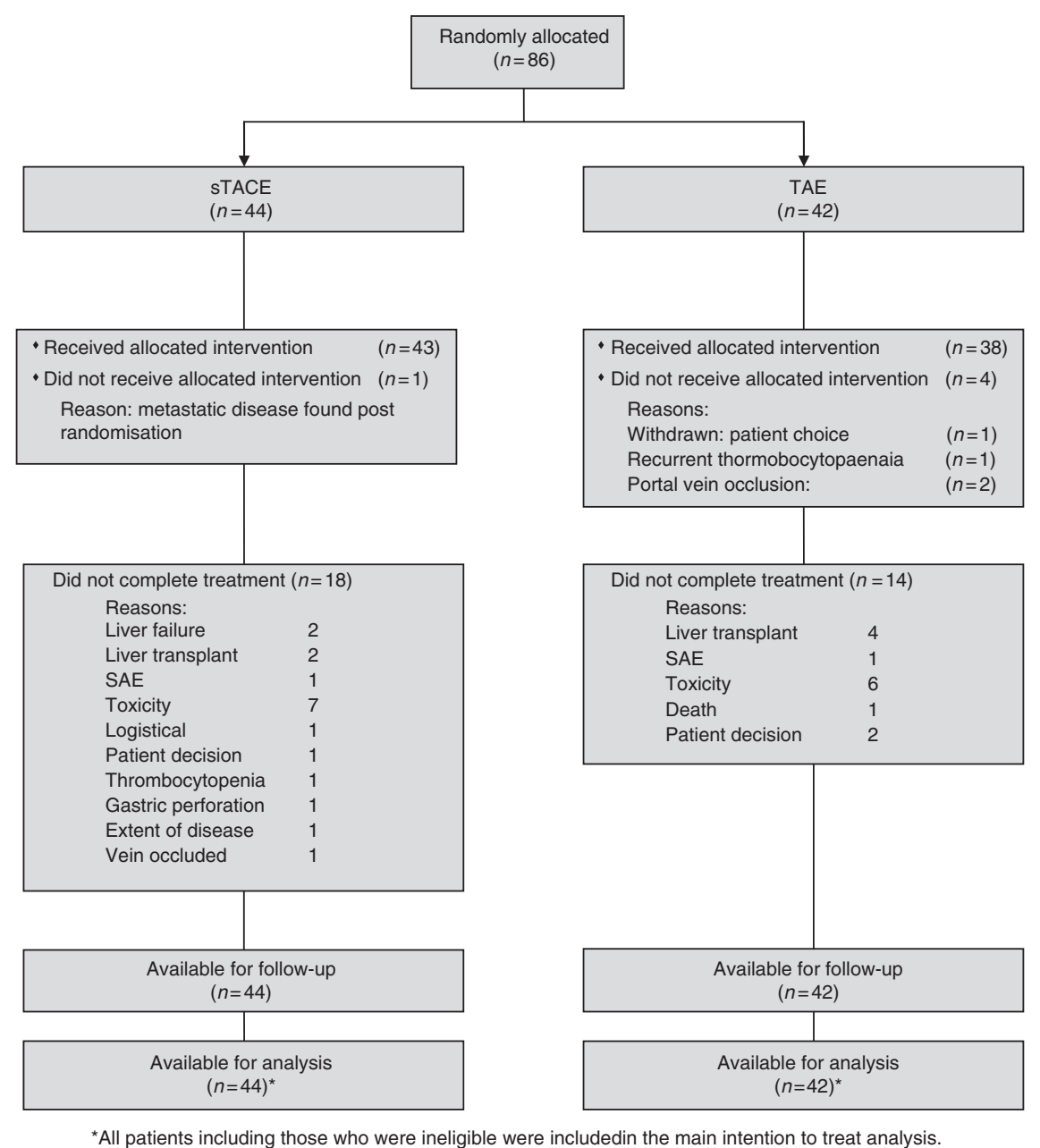

Figure 1. Consort diagram. 


\section{RESULTS}

Patients and treatment. A total of 86 patients were recruited from two sites; the Royal Free Hospital and University College London Hospital, between December 2003 and November 2009. Patients were randomised to TAE $(n=42)$ or sTACE $(n=44)$ with 38 and 43 patients, respectively, receiving the allocated intervention (Figure 1).

Baseline characteristics are shown in Table 1. Patients were well matched in terms of key prognostic criteria including Child Pugh score, focality, performance status and aetiology of liver disease. Overall 82.6\% had Child Pugh A cirrhosis, 67.4\% were ECOG performance status $0,67.4 \%$ had multifocal disease, $39.5 \%$ were BCLC stage B and $31.4 \%$ stage C due to PS $>0$.

In the TAE arm, $57.1 \%$ of patients received all three planned treatments compared with $56.8 \%$ in the sTACE arm and at least two treatments were delivered to $64.3 \%$ and $75.0 \%$ of patients in the two arms, respectively. Two patients in the sTACE arm received only chemotherapy and were not embolised for one or two of their treatments due to development of portal vein occlusion and extent of bilobar disease, respectively. There was no significant difference between the arms with respect to the number of treatments patients received $(P=0.26)$. The most common reason that patients stopped treatment early was adverse events or toxicity. However the number of patients stopping early was similar in each arm; $7(16.7 \%)$ in the TAE and $8(18.2 \%)$ in the sTACE arm. Treatments were delivered in a timely manner with over $84.3 \%$ of treatments in the TAE arm being given within $48 \mathrm{~h}$ of the planned procedure and $84.8 \%$ in the sTACE arm. In $66.8 \%$ of cases, particle embolisation was unilobar and there were no dose reductions required for cisplatin. The median length of hospital stay for all treatments was 2 days range (1-41 days).

Toxicity and safety. For TAE and sTACE, the most common grade $3 / 4$ non-haematological toxicity was grade 3 pain occurring in $7(18.4 \%)$ and $10(23.3 \%)$ patients (Table 2). One patient in the TAE arm and two in the sTACE arm had grade 4 pain. Two patients receiving sTACE developed a liver abscess. The most common biochemical disturbance was grade 3 elevation of gammaglutamyl transpeptidase occurring in $9(23.7 \%)$ and 17 (39.5\%) patients ( 1 patient in the TAE arm had a grade 4 elevation) and grade 3 hyperbilirubinaemia in $5(13.2 \%)$ and $4(9.3 \%)$ patients. One patient in the TAE arm and two in the sTACE arm experienced grade 4 hyperbilirubinaemia. Within the sTACE group, myelosuppression was very uncommon with only one patient experiencing grade 3 neutropenia. Overall grade $3 / 4$ toxicity occurred in $63.5 \%$ and $83.7 \%$ in the TAE and sTACE groups, respectively $(P=0.019)$. There were two deaths that were classified as treatment-related. One patient in the sTACE arm completed all three planned treatments and died 43 days later after his third sTACE from decompensated liver disease. The second patient was in the TAE arm and developed renal and liver failure following the first treatment and died 17 days after the TAE procedure.

Efficacy. For patients who had at least one treatment, the end-oftreatment response rates according to RECIST 1.0 were $13.2 \%$ and 32.6\% $(P=0.04)$ for TAE and sTACE, respectively. Four patients in each group achieved a partial response (PR) on further followup giving a best overall response rate (PR) of $23.7 \%$ and $41.9 \%$. There were no complete responses (CR) by RECIST and progressive disease (PD) occurred in $26.3 \%$ and $7 \%$. Applying the mRECIST criteria, the overall response rate (CR and PR) was $47.3 \%$ and $67.4 \%$, respectively (Table 3 ).

All 86 patients were included in the final survival analysis, which was conducted 15.6 months after the last patient was randomised. At the time of the analysis, the median follow-up was

\section{Table 1. Baseline characteristics}

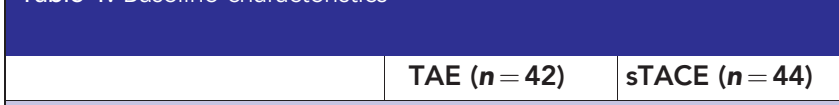

Age

\begin{tabular}{|l|c|r|}
\hline Median (range) & $62.6(30.7,85.2)$ & $63.2(44.1,79.3)$ \\
\hline Gender & $35(83.3)$ & $39(88.6)$ \\
\hline Male & $7(16.7)$ & $5(11.4)$ \\
Female &
\end{tabular}

Child Pugh status

\begin{tabular}{|l|r|r|}
\hline A & $33(78.6)$ & $38(86.4)$ \\
B & $9(21.4)$ & $6(13.6)$ \\
\hline
\end{tabular}

\section{ECOG PS}

\begin{tabular}{|l|r|r|}
\hline 0 & $27(64.3)$ & $31(70.5)$ \\
1 & $9(21.4)$ & $8(18.2)$ \\
2 & $6(14.3)$ & $5(11.4)$ \\
\hline
\end{tabular}

Ascites

Absent

Slight

Moderate

Unknown

$38(90.5)$

$3(7.1)$

$1(2.4)$

0

35 (79.5)

7 (15.9)

$1(2.3)$

$1(2.3)$

Hepatitis B

\begin{tabular}{|l|c|c|}
\hline Absent & $37(88.1)$ & $34(77.3)$ \\
Present & $5(11.9)$ & $9(20.5)$ \\
Unknown & 0 & $1(2.3)$
\end{tabular}

Hepatitis C

\begin{tabular}{|l|c|c|}
\hline Absent & $23(54.8)$ & $27(61.4)$ \\
Present & $19(45.2)$ & $16(36.4)$ \\
Unknown & $0(0.0)$ & $1(2.3)$
\end{tabular}

Alcohol disease

\begin{tabular}{|l|c|c|}
\hline Absent & $37(88.1)$ & $30(68.2)$ \\
Present & $5(11.9)$ & $13(29.5)$ \\
Unknown & 0 & $1(2.3)$ \\
\hline
\end{tabular}

Disease focality

Unifocal

Multifocal: 2 nodules

Multifocal: $>2$ nodules

Diffuse

Multifocal: number unknown

$13(31.0)$
$7(16.7)$
$16(38.1)$
$3(7.1)$
$3(7.1)$

$15(34.1)$

$12(27.3)$

$14(31.8)$

$2(4.5)$

$1(2.3)$

Liver volume occupied by tumour

\begin{tabular}{|l|c|c|}
\hline$\leqslant 50 \%$ & $36(85.7)$ & $36(81.8)$ \\
$>50 \%$ & $2(4.8)$ & 0 \\
Unknown & $4(9.5)$ & $8(18.2)$ \\
\hline
\end{tabular}

Disease extent

\begin{tabular}{|l|r|r|}
\hline Unilobar & $21(50.0)$ & $21(47.7)$ \\
Bilobar & $16(38.1)$ & $15(34.1)$ \\
Unknown & $5(11.9)$ & $8(18.2)$
\end{tabular}

Baseline AFP $\left(\mathrm{ng} \mathrm{ml}^{-1}\right)$

\begin{tabular}{|l|l|l|}
\hline$\leqslant 400$ & $32(76.2)$ & $33(75.0)$ \\
$>400$ & $10(23.8)$ & $11(25.0)$ \\
\hline
\end{tabular}

Size of largest nodule

\begin{tabular}{|l|l|r|}
\hline$\leqslant 7 \mathrm{~cm}$ & $28(66.7)$ & $37(84.1)$ \\
$>7 \mathrm{~cm}$ & $14(33.3)$ & $7(15.9)$
\end{tabular}




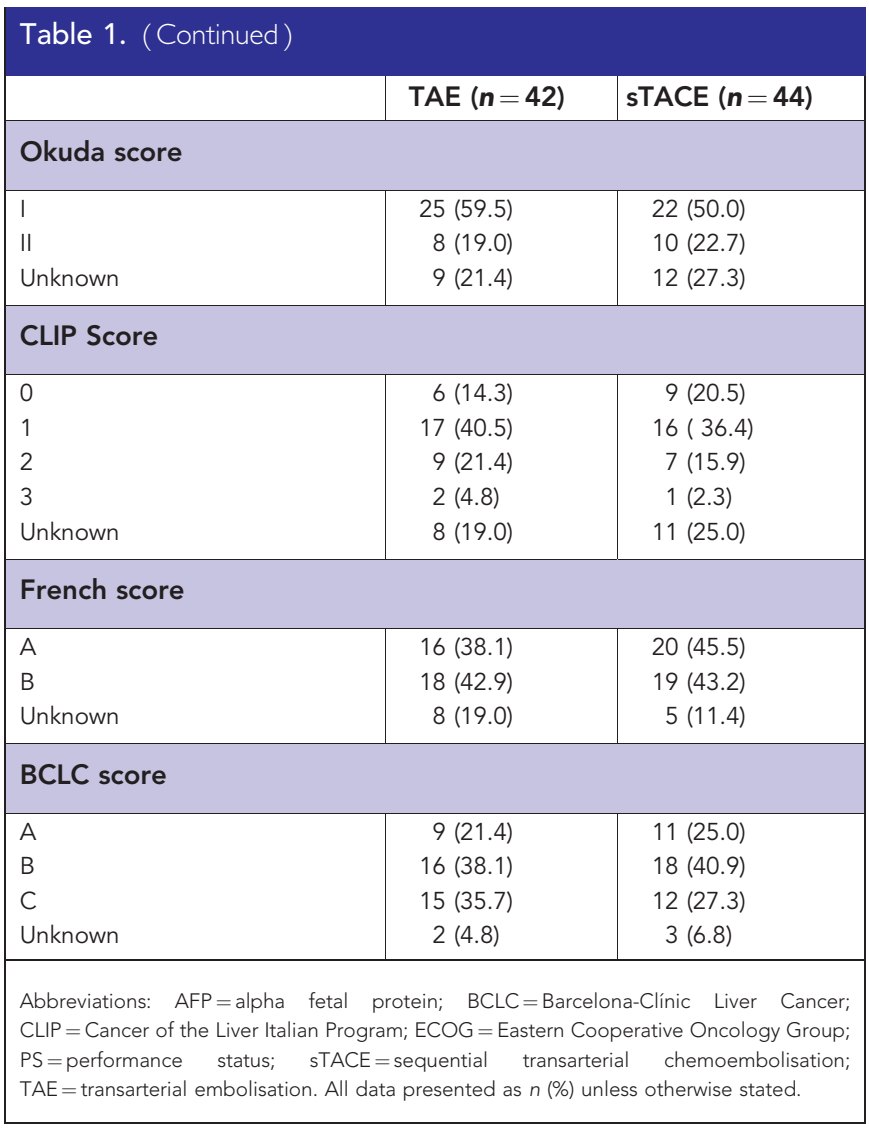

24.0 months (range: $0.03-56.0$ ), $54.7 \%$ patients had died and $68.6 \%$ had met the progression-free survival endpoint. The cause of death in $83.0 \%$ of cases was progressive hepatocellular carcinoma. According to the intention-to-treat analysis there was no difference in survival between the TAE and the sTACE group (HR: 0.91, 95\% CI: $0.51-1.62, P=0.74)$ with a median survival of 17.3 and 16.3 months for each group, respectively (Figure $2 \mathrm{~B}$ ).

The 1-year survival rates were $68 \%$ (95\% CI; $50-81)$ and $64 \%$ (95\% CI; 45-78), and the 2-year survival rates were 39\% (95\% CI $22-56)$ and $32 \%$ (95\% CI 15-50) for TAE and TACE, respectively. There was also no difference in progression-free survival time between the two arms (HR: $0.87,95 \%$ CI: $0.52-1.45, P=0.59$ ) with a median of 7.2 months for TAE and 7.5 months for TACE Figure 2A).

Four patients in the TAE group and two in the TACE group underwent liver transplantation during the course of the planned treatment and a further five and eight, respectively, underwent transplantation during the follow-up period. When the transplanted patients were censored at the time of transplant the 1-year, 2 -year and median overall survival was; $64.5 \%$ (44.8-78.8), $32.7 \%$ (16.0-50.5) and 16.2 months for TAE and 61.9\% (42.4-76.5), $25.4 \%(9.8-44.7)$ and 16.0 for sTACE.

Quality of life. Quality of life data was collected at baseline, $72 \mathrm{~h}$ pre-randomisation, 2-4 weeks after treatment completion and at the 3 and 6 month follow-up appointments using the EORTC QLQ-C30 questionnaire. Data for the later time points was sparse so the decision was taken to analyse only the change in scores from baseline to post-treatment. This gave 33 assessable patients. None of the scores showed any significant difference between the arms suggesting that the addition of chemotherapy did not lead to deterioration in quality of life compared with bland embolisation (Table 4).

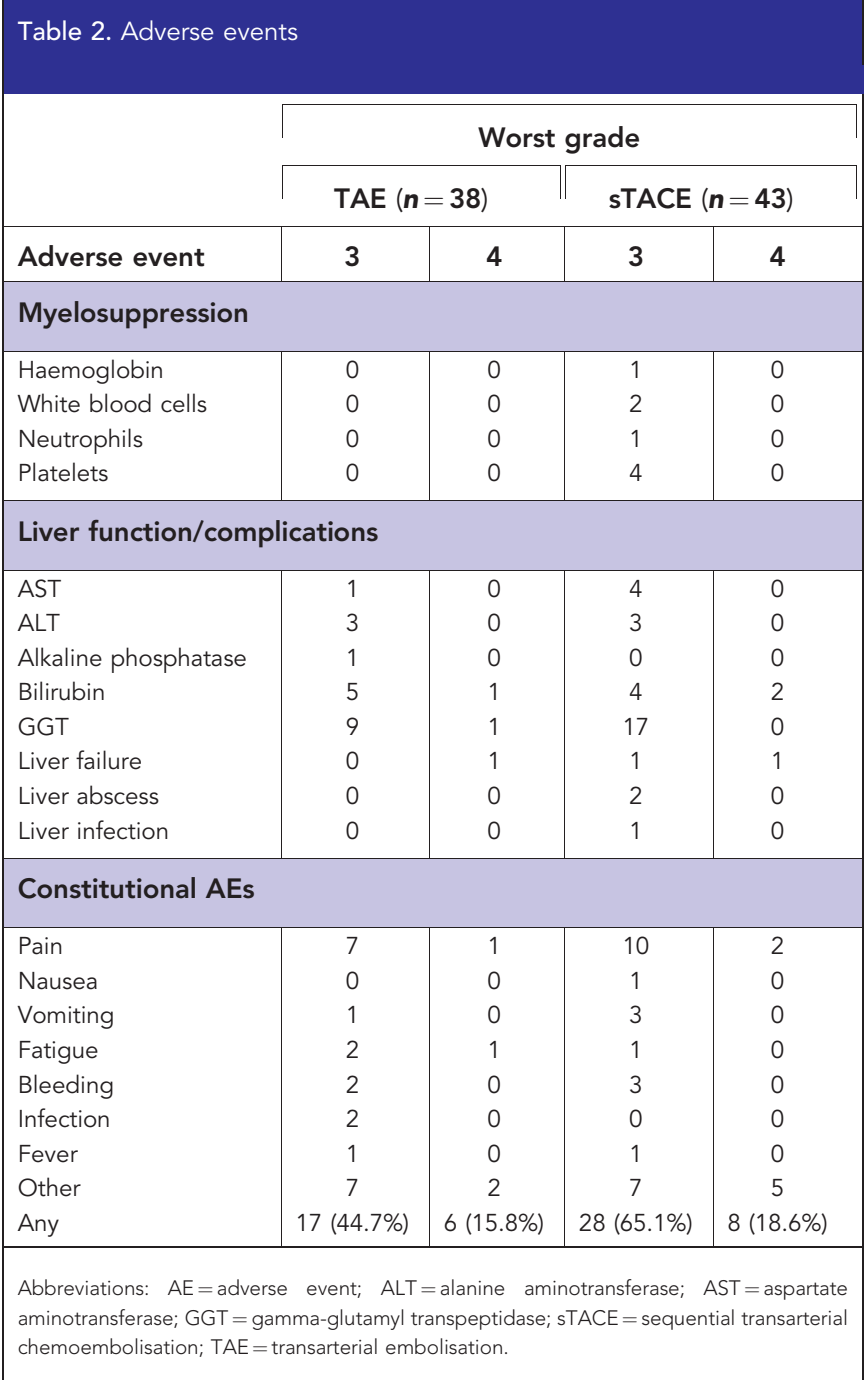

Meta-analysis of trials comparing TAE $v$ s TACE. We identified five eligible trials including the present one, of which three, including the current study, had a mean follow-up of 2 years (Kawai et al, 1992; Llovet et al, 2002) and the remaining two of 1 year (Chang et al, 1994; Malagari et al, 2010). We wrote to the authors of one study to obtain information not included in the article (Chang et al, 1994). Transarterial chemoembolisation was performed with doxorubicin and lipiodol in two studies (Kawai et al, 1992; Llovet et al, 2002), with doxorubicin-loaded DEBTACE in one (Malagari et al, 2010) and cisplatin in our study and one other (Chang et al, 1994). TAE was performed with a variety of materials: lipiodol and gelfoam (Kawai et al, 1992; Chang et al, 1994), gelfoam alone (Llovet et al, 2002), BeadBlocks (Malagari et al, 2010) and PVA particles in our study. As shown in Figure 3, there was no significant difference in mortality between the two modalities (RR 1.09, 95\% CI 0.84-1.41, $P=0.13$ ) and although there was some heterogeneity between studies $\left(I^{2}=44 \%\right)$, it was not significant $(P=0.13)$.

\section{DISCUSSION}

According to the most recent EASL-EORTC practice guidelines, chemoembolisation is recommended for patients with BCLC stage $\mathrm{B}$, multinodular asymptomatic tumours without vascular invasion 
Table 3. Response rates according to RECIST 1.0 and mRECIST

\begin{tabular}{|c|c|c|}
\hline Response & TAE $\mathbf{N}=38(\%)$ & sTACE $\mathbf{N}=\mathbf{4 3}(\%)$ \\
\hline \multicolumn{3}{|c|}{ Response (EOT) } \\
\hline $\begin{array}{l}\mathrm{PR} \\
\mathrm{SD} \\
\mathrm{PD} \\
\text { Missing } \\
{ }^{*} P=0.04\end{array}$ & $\begin{array}{r}5(13.2) \\
18(47.4) \\
10(26.3) \\
5(13.2)\end{array}$ & $\begin{array}{c}14(32.6) \\
21(48.8) \\
3(7.0) \\
5(11.6)\end{array}$ \\
\hline \multicolumn{3}{|c|}{ Best response } \\
\hline $\begin{array}{l}P R \\
S D \\
P D \\
\text { Missing } \\
{ }^{*} P=0.08\end{array}$ & $\begin{array}{r}9(23.7) \\
15(39.5) \\
10(26.3) \\
4(10.5)\end{array}$ & $\begin{array}{c}18(41.9) \\
17(38.5) \\
3(7.0) \\
5(11.6)\end{array}$ \\
\hline \multicolumn{3}{|c|}{ mRECIST response (EOT) } \\
\hline $\begin{array}{l}\text { CR } \\
P R \\
S D \\
P D \\
\text { Missing } \\
\star P=0.068\end{array}$ & $\begin{array}{r}7(18.4) \\
11(28.9) \\
4(10.5) \\
6(15.8) \\
10(26.3)\end{array}$ & $\begin{aligned} 13 & (30.2) \\
16 & (37.2) \\
6 & (14.0) \\
2 & (4.7) \\
6 & (14.0)\end{aligned}$ \\
\hline \multicolumn{3}{|c|}{$\begin{array}{l}\text { Abbreviations: } C R=\text { complete response; } E O T=\text { end of treatment; } P D=\text { progressive } \\
\text { disease; } P R=\text { partial response; } s T A C E=C \text { Chi-squared response }(C R, P R) \text { vs no response } \\
(S D, P D, \text { missing). }\end{array}$} \\
\hline
\end{tabular}

A

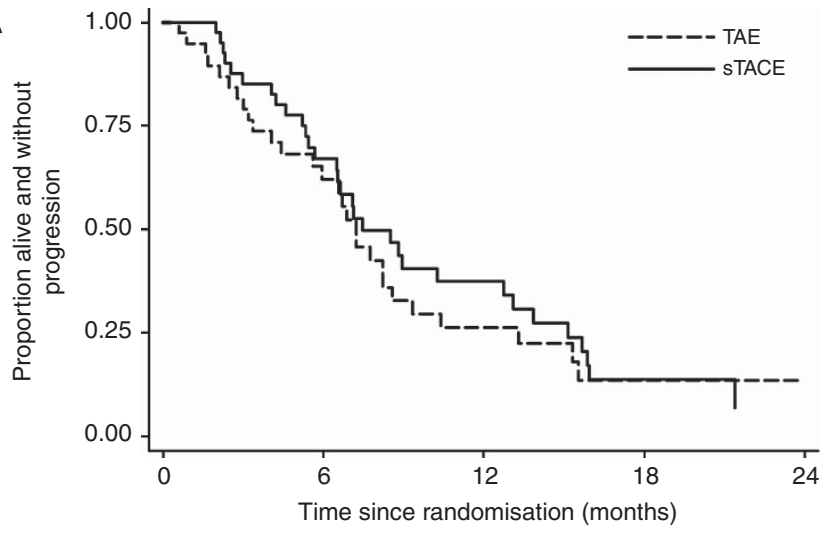

Number at risk
TAE 42

STACE 44
20

25

\section{7}

3

2

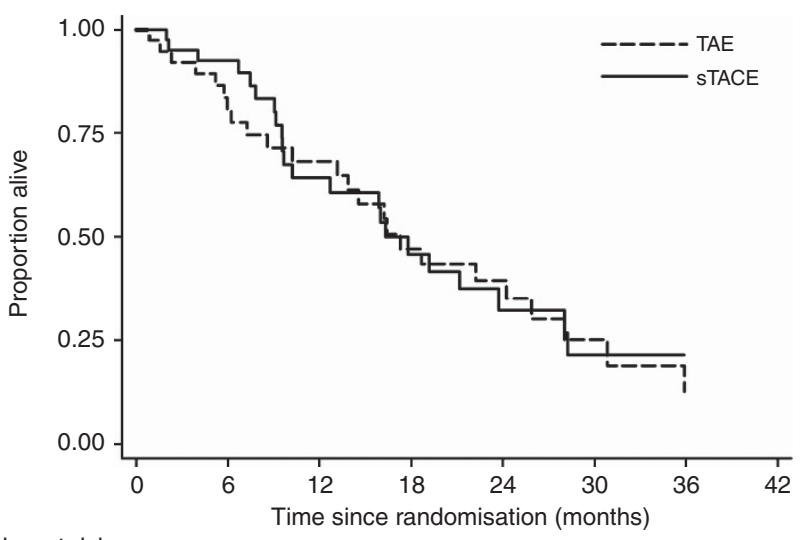

B

Number at risk

$\begin{array}{rlllllll}\text { TAE } 42 & 27 & 20 & 13 & 9 & 5 & 2 & 0 \\ \text { STACE 44 } & 35 & 18 & 11 & 6 & 4 & 3 & 1\end{array}$

Figure 2. (A) Progression-free survival. (B) overall survival.
Table 4. Comparison of quality of life parameters between sTACE- and TAE-treated patients

\begin{tabular}{|c|c|c|}
\hline Score & $\begin{array}{l}\text { Mean difference adjusted } \\
\text { for baseline score }(99 \% \mathrm{Cl})\end{array}$ & $\boldsymbol{P}$-value \\
\hline Global health status/ $\mathrm{QOL}^{\mathrm{a}}$ & $-3.18(-28.63,22.28)$ & 0.73 \\
\hline
\end{tabular}

\section{Functional scales ${ }^{a}$}

Physical functioning

Role functioning

Emotional functioning

Cognitive functioning

Social functioning

\begin{tabular}{l|l}
$3.33(-20.89,27.55)$ & 0.71 \\
$0.24(-37.51,37.03)$ & 0.99 \\
$2.12(-28.21,23.97)$ & 0.82 \\
$5.41(-30.54,19.72)$ & 0.56 \\
$1.28(-34.46,37.03)$ & 0.92
\end{tabular}

Symptom scales ${ }^{b, c}$

Fatigue

Nausea and vomiting

Pain

Dyspnoea

Insomnia

Appetite loss

Constipation

Financial difficulties

$10.76(-14.21,35.73)$

-4.66 ( $-23.58,14.27)$

$-2.33(-27.77,23.11)$

$-12.54(-41.93,16.86)$

$1.08(-28.95,31.12)$

$-0.67(-32.3,30.93)$

$0.38(-20.71,21.47)$

$-14.34(-37.02,8.34)$

Abbreviations: $\mathrm{Cl}=$ confidence interval; $\mathrm{QOL}=$ quality of life; sTACE = sequential transar terial chemoembolisation; $\mathrm{TAE}=$ transarterial embolisation. Higher mean differences indicate patients in the TACE arm have higher scores than those in the TAE arm.

${ }^{{ }^{a}}$ Higher scores are positive; they indicate better functioning and $\mathrm{QOL}$.

$\mathbf{b}_{\text {Information on diarrhoea was also collected but all but five patients at baseline and four }}$ post treatment had a score of 0 .

${ }^{c_{H}}$ igher scores are negative: they indicate worse symptoms.

or extra-hepatic spread (European Association For The Study Of The Liver and European Organisation For Research And Treatment Of Cancer, 2012). However, the optimal method or schedule remains undefined and the contribution of chemotherapy remains uncertain (Marelli et al, 2007). In our trial, we evaluated a novel TACE protocol (sTACE) designed to optimise the effect of chemotherapy and overcome some of the theoretical flaws in the conventional protocols. The protocol proved to be feasible and tolerable in the majority of patients although grade $3 / 4$ toxicity was increased in the sTACE arm compared with the TAE arm. The response rates according to both RECIST 1.0 and mRECIST tended to be higher in the sTACE arm and we have previously shown that end-of-treatment response according to mRECIST is an independent factor predicting better survival (Gillmore et al, 2011). However, in this trial, the differences in the mRECIST response between the two arms did not reach significance nor was there evidence that sTACE improved survival compared with TAE.

The patient population included in our study had characteristics similar to those reported in the two previous studies reporting positive outcome with TACE (Llovet et al, 2002; Lo et al, 2002) and the majority of patients were BCLC stage B. Those patients with BCLC stage C disease were classified as such on the basis of their performance status only. The survival outcome in our study was also very similar to that reported by Lo et al (2002), who also used cisplatin-based TACE and reported a 1-year survival of 57\% and 2 -year survival of $31 \%$, which compares with $66 \%$ and $36 \%$ in our trial (Lo et al, 2002). The recent EASL guidelines (European Association For The Study Of The Liver and European Organisation For Research And Treatment Of Cancer, 2012) acknowledge the wide range of survival outcomes reported for TACE-treated patients, which varies between 13 months in multicentre prospective trials (Doffoel et al, 2008) to 48 months in single centres (Burrel et al, 2012). Within the BCLC B classification, there is no limit on tumour size, number of tumours 


\begin{tabular}{|c|c|c|c|c|c|c|c|c|}
\hline \multirow[b]{2}{*}{ Study or subgroup } & \multicolumn{2}{|c|}{ TACE } & \multicolumn{2}{|c|}{ TAE } & \multicolumn{2}{|r|}{ Risk ratio } & \multicolumn{2}{|l|}{ Risk ratio } \\
\hline & Events & Total & Events & Total & Weight & $\mathrm{M}-\mathrm{H}$, Random, $95 \% \mathrm{Cl}$ & $\mathrm{M}-\mathrm{H}$, Random, $95 \% \mathrm{Cl}$ & \\
\hline Chang et al (1994) & 14 & 22 & 9 & 24 & $13.5 \%$ & $1.70(0.93,3.11)$ & - & \\
\hline Kawai et al (1992) & 82 & 148 & 62 & 141 & $35.1 \%$ & $1.26(1.00,1.60)$ & & \\
\hline Llovet et al (2002) & 21 & 40 & 25 & 37 & $24.8 \%$ & $0.78(0.54,1.12)$ & - & \\
\hline Malagari et al (2010) & 6 & 41 & 6 & 43 & $5.5 \%$ & $1.05(0.37,2.99)$ & & \\
\hline Meyer et al (this study) & 21 & 44 & 21 & 42 & $21.0 \%$ & $0.95(0.62,1.47)$ & & \\
\hline Total $(95 \% \mathrm{Cl})$ & & 295 & & 287 & $100.0 \%$ & $1.09(0.84,1.41)$ & & \\
\hline Total events & 144 & & 123 & & & & & \\
\hline $\begin{array}{l}\text { Heterogeneity: } \tau^{2}=0.0 \\
\text { Test for overall effect: } Z\end{array}$ & $\begin{array}{l}4 ; \chi^{2}=7 \\
=0.62\end{array}$ & $\begin{array}{l}09, \mathrm{df} \\
P=0 .\end{array}$ & $=4(P=$ & .13); & $=44 \%$ & 0.0 & 0.1 & 100 \\
\hline
\end{tabular}

Figure 3. Forrest plot for survival outcomes following TACE or TAE alone in patients with hepatocellular carcinoma in the five existing randomised controlled trials to date.

or level of AFP, and both child A and B patients are included. Hence, the prognostic spectrum within this group is wide and patient selection is likely to be an important determinant of survival.

The anticipated benefit of adding chemotherapy to TACE assumes that hepatocellular is sufficiently chemosensitive to improve survival over and above that obtained by TAE alone. However, systemic chemotherapy has been used for over 30 years in the treatment of HCC and is associated with response rates of less than $10 \%$ in large randomised trials (Burroughs et al, 2004; Asghar and Meyer, 2012). Furthermore, the survival benefit is uncertain, as there have been no large randomised trials comparing chemotherapy vs best supportive care. Given that transarterial embolisation alone is highly effective in inducing tumour necrosis (Marelli et al, 2007), it may be difficult to detect any improvement by adding local chemotherapy, which has limited efficacy as a single agent. In our study, we did not use lipiodol and we have previously reported the lack of evidence for its use (Marelli et al, 2007). As chemotherapy is hydrophilic, it rapidly moves into the systemic circulation when co-administered with lipiodol, and pharmacokinetic studies demonstrate that area under the concentration-time curve for doxorubicin is the same whether administered as bolus injection or as an emulsion with lipiodol (Johnson et al, 1991; Dodds et al, 1996). The recent introduction of DEBs allow the delivery of high local concentrations of doxorubicin with reduced systemic AUC (Varela et al, 2007), yet trials comparing DEB-TACE with conventional lipiodol-based TACE (cTACE) do not show an improved response rate, although the toxicity attributable to chemotherapy is substantially reduced (Lammer et al, 2010; Malagari et al, 2010). Moreover, the results of a randomised trial comparing DEB-TACE with bead-alone TAE has recently been reported and published in abstract form. No significant difference in response rate or survival was found (Brown et al, 2012).

As our trial was discontinued at phase II, we were not able to assess the primary survival endpoint of the phase III component. However, some evidence regarding survival has been provided by an update of our previous meta-analysis (Marelli et al, 2007) in which we have included data from the current trial and another recently published study (Malagari et al, 2010), which increases the total number of patients included to 582 from five randomised trials. No significant difference was found and there is therefore no current evidence that TACE using any method is superior TAE alone. A definitive answer to this question can only be obtained by adequately powered randomised phase III trials.

Despite TAE or TACE, patients relapse at the treated site, or at new hepatic sites or in extra-hepatic locations. Efforts should therefore focus on reducing the rate of relapse or progression by the addition of effective systemic therapy. The current standard of care for advanced disease is sorafenib, which acts partly through the inhibition of signalling through VEGFR and PDGFR, both known to be important for tumour angiogenesis. Tumour embolisation results in hypoxia and the transcription of genes such as VEGF leads to angiogenesis and this may contribute to tumour re-growth. Hence the concurrent administration of sorafenib with intra-arterial embolisation may prevent relapse of treated disease and growth of new or metastatic tumours. This combination has been shown to be safe in phase I and II trials (Dufour et al, 2010; Pawlik et al, 2011; Lencioni R et al, 2012) and a randomised phase II trial has also been reported (Lencioni $\mathrm{R}$ et al, 2012). Two randomised phase III trials; TACE 2 (NCT01324076) and ECOG1208 (NCT01004978), continue to accrue and a number of other systemic agents are also being evaluated in this setting.

\section{ACKNOWLEDGEMENTS}

This work was in part supported by the National Institute of Health Research (NIHR), Experimental Cancer Medicine Centre Network (ECMC). TM is partly funded by UCLH/UCL Department of Health's NIHR Biomedical Research Centres funding scheme.

\section{REFERENCES}

Asghar U, Meyer T (2012) Are there opportunities for chemotherapy in the treatment of hepatocellular cancer? J Hepatol 56(3): 686-695.

Brown KT, Gonen M, Gian Do K, Covey AM, Getrajdman GI, Zhao B, Sofocleous CT, Beattie C, DeMatteo RP, Solomon SB, Abou-Alfa GK (2012) A randomized single blind controlled trial of beads versus doxorubicin-eluting beads for arterial embolization of hepatocellular carcinoma (HCC). J Clin Oncol 30(suppl 34): abstract 143.

Bruix J, Sherman M, Llovet JM, Beaugrand M, Lencioni R, Burroughs AK, Christensen E, Pagliaro L, Colombo M, Rodes J (2001) Clinical management of hepatocellular carcinoma. Conclusions of the Barcelona2000 EASL conference. European Association for the Study of the Liver. J Hepatol 35(3): 421-430.

Burrel M, Reig M, Forner A, Barrufet M, de Lope CR, Tremosini S, Ayuso C, Llovet JM, Real MI, Bruix J (2012) Survival of patients with hepatocellular carcinoma treated by transarterial chemoembolisation (TACE) using drug eluting beads. Implications for clinical practice and trial design. J Hepatol 56(6): 1330-1335.

Burroughs A, Hochhauser D, Meyer T (2004) Systemic treatment and liver transplantation for hepatocellular carcinoma: two ends of the therapeutic spectrum. Lancet Oncol 5(7): 409-418.

Chang JM, Tzeng WS, Pan HB, Yang CF, Lai KH (1994) Transcatheter arterial embolization with or without cisplatin treatment of hepatocellular carcinoma. A randomized controlled study. Cancer 74(9): 2449-2453. 
CLIP Investigators (1998) A new prognostic system for hepatocellular carcinoma: a retrospective study of 435 patients: the Cancer of the Liver Italian Program (CLIP) investigators. Hepatology 28(3): 751-755.

Dodds HM, Walpole ET, Rivory LP, Strong RW, Pond SM (1996) Disposition of epirubicin after intraarterial administration in Lipiodol to patients with hepatocellular carcinoma. Ther Drug Monit 18(5): 537-543.

Doffoel M, Bonnetain F, Bouche O, Vetter D, Abergel A, Fratte S, Grange JD, Stremsdoerfer N, Blanchi A, Bronowicki JP, Caroli-Bosc FX, Causse X, Masskouri F, Rougier P, Bedenne L (2008) Multicentre randomised phase III trial comparing Tamoxifen alone or with Transarterial Lipiodol Chemoembolisation for unresectable hepatocellular carcinoma in cirrhotic patients (Federation Francophone de Cancerologie Digestive 9402). Eur J Cancer 44(4): 528-538.

Dufour JF, Hoppe H, Heim MH, Helbling B, Maurhofer O, Szucs-Farkas Z, Kickuth R, Borner M, Candinas D, Saar B (2010) Continuous administration of sorafenib in combination with transarterial chemoembolization in patients with hepatocellular carcinoma: results of a phase I study. Oncologist 15(11): 1198-1204.

European Association For The Study Of The Liver, European Organisation For Research And Treatment Of Cancer (2012) EASL-EORTC Clinical Practice Guidelines: Management of hepatocellular carcinoma. J Hepatol 56(4): 908-943.

Forner A, Llovet JM, Bruix J (2012) Chemoembolization for intermediate HCC: is there proof of survival benefit? J Hepatol 56(4): 984-986.

Gillmore R, Stuart S, Kirkwood A, Hameeduddin A, Woodward N, Burroughs AK, Meyer T (2011) EASL and mRECIST responses are independent prognostic factors for survival in hepatocellular cancer patients treated with transarterial embolization. J Hepatol 55(6): 1309-1316.

Johnson PJ, Kalayci C, Dobbs N, Raby N, Metivier EM, Summers L, Harper P, Williams R (1991) Pharmacokinetics and toxicity of intraarterial adriamycin for hepatocellular carcinoma: effect of coadministration of lipiodol. J Hepatol 13(1): 120-127.

Kawai S, Okamura J, Ogawa M, Ohashi Y, Tani M, Inoue J, Kawarada Y, Kusano M, Kubo Y, Kuroda C (1992) Prospective and randomized clinical trial for the treatment of hepatocellular carcinoma-a comparison of lipiodol-transcatheter arterial embolization with and without adriamycin (first cooperative study). The Cooperative Study Group for Liver Cancer Treatment of Japan. Cancer Chemother Pharmacol 31(Suppl): S1-S6.

Lammer J, Malagari K, Vogl T, Pilleul F, Denys A, Watkinson A, Pitton M, Sergent G, Pfammatter T, Terraz S, Benhamou Y, Avajon Y, Gruenberger T, Pomoni M, Langenberger H, Schuchmann M, Dumortier J, Mueller C, Chevallier P, Lencioni R (2010) Prospective randomized study of doxorubicin-eluting-bead embolization in the treatment of hepatocellular carcinoma: results of the PRECISION V study. Cardiovasc Intervent Radiol 33(1): 41-52.

Lencioni R, Llovet JM (2010) Modified RECIST (mRECIST) assessment for hepatocellular carcinoma. Semin Liver Dis 30(1): 52-60.

Lencioni R, Llovet JM, Han G, Tak W-Y, Yang J, Leberre M-A, Niu W, Nicholson K, Meinhardt G, Bruix J (2012) Sorafenib or placebo in combination with transarterial chemoembolization (TACE) with doxorubicin-eluting beads (DEBDOX) for intermediate-stage hepatocellular carcinoma (HCC): Phase II, randomized, double-blind SPACE trial. J Clin Oncol 30(suppl 4): abstract 154.

Llovet JM, Bru C, Bruix J (1999) Prognosis of hepatocellular carcinoma: the BCLC staging classification. Semin Liver Dis 19(3): 329-338.

Llovet JM, Bruix J (2003) Systematic review of randomized trials for unresectable hepatocellular carcinoma: Chemoembolization improves survival. Hepatology 37(2): 429-442.

Llovet JM, Real MI, Montana X, Planas R, Coll S, Aponte J, Ayuso C, Sala M, Muchart J, Sola R, Rodes J, Bruix J (2002) Arterial embolisation or chemoembolisation versus symptomatic treatment in patients with unresectable hepatocellular carcinoma: a randomised controlled trial. Lancet 359(9319): 1734-1739.

Lo CM, Ngan H, Tso WK, Liu CL, Lam CM, Poon RT, Fan ST, Wong J (2002) Randomized controlled trial of transarterial lipiodol chemoembolization for unresectable hepatocellular carcinoma. Hepatology 35(5): 1164-1171.

Malagari K, Pomoni M, Kelekis A, Pomoni A, Dourakis S, Spyridopoulos T, Moschouris H, Emmanouil E, Rizos S, Kelekis D (2010) Prospective randomized comparison of chemoembolization with doxorubicin-eluting beads and bland embolization with BeadBlock for hepatocellular carcinoma. Cardiovasc Intervent Radiol 33(3): 541-551.

Marelli L, Stigliano R, Triantos C, Senzolo M, Cholongitas E, Davies N, Tibballs J, Meyer T, Patch DW, Burroughs AK (2007) Transarterial therapy for hepatocellular carcinoma: which technique is more effective? A systematic review of cohort and randomized studies. Cardiovasc Intervent Radiol 30(1): 6-25.

Moher D, Liberati A, Tetzlaff J, Altman DG (2009) Preferred reporting items for systematic reviews and meta-analyses: the PRISMA statement. BMJ 339: b2535.

Okuda K, Ohtsuki T, Obata H, Tomimatsu M, Okazaki N, Hasegawa H, Nakajima Y, Ohnishi K (1985) Natural history of hepatocellular carcinoma and prognosis in relation to treatment. Study of 850 patients. Cancer 56(4): 918-928.

Oliveri RS, Wetterslev J, Gluud C (2011) Transarterial (chemo)embolisation for unresectable hepatocellular carcinoma. Cochrane Database Syst Rev (3): CD004787.

Parkin DM, Bray F, Ferlay J, Pisani P (2005) Global cancer statistics, 2002. CA Cancer J Clin 55(2): 74-108.

Pawlik TM, Reyes DK, Cosgrove D, Kamel IR, Bhagat N, Geschwind JF (2011) Phase II trial of sorafenib combined with concurrent transarterial chemoembolization with drug-eluting beads for hepatocellular carcinoma. J Clin Oncol 29(30): 3960-3967.

Ray Jr CE, Haskal ZJ, Geschwind JF, Funaki BS (2011) The use of transarterial chemoembolization in the treatment of unresectable hepatocellular carcinoma: a response to the Cochrane Collaboration review of 2011. J Vasc Interv Radiol 22(12): 1693-1696.

Schulz KF, Altman DG, Moher D (2010) CONSORT 2010 statement: updated guidelines for reporting parallel group randomised trials. BMJ 340: $\mathrm{c} 332$.

Tak E, Lee S, Lee J, Rashid MA, Kim YW, Park JH, Park WS, Shokat KM, Ha J, Kim SS (2011) Human carbonyl reductase 1 upregulated by hypoxia renders resistance to apoptosis in hepatocellular carcinoma cells. J Hepatol 54(2): 328-339.

Therasse P, Arbuck SG, Eisenhauer EA, Wanders J, Kaplan RS, Rubinstein L, Verweij J, Van GM, van Oosterom AT, Christian MC, Gwyther SG (2000) New guidelines to evaluate the response to treatment in solid tumors. European Organization for Research and Treatment of Cancer, National Cancer Institute of the United States, National Cancer Institute of Canada. J Natl Cancer Inst 92(3): 205-216.

Varela M, Real MI, Burrel M, Forner A, Sala M, Brunet M, Ayuso C, Castells L, Montana X, Llovet JM, Bruix J (2007) Chemoembolization of hepatocellular carcinoma with drug eluting beads: efficacy and doxorubicin pharmacokinetics. J Hepatol 46(3): 474-481.

This work is published under the standard license to publish agreement. After 12 months the work will become freely available and the license terms will switch to a Creative Commons AttributionNonCommercial-Share Alike 3.0 Unported License. 